\title{
TEXTURE ANALYSIS IN PERFUSION IMAGES OF PROSTATE CANCER-A CASE STUDY
}

\author{
JACEK ŚMIETAŃSKI*, RYSZARD TADEUSIEWICZ **, ELŻBIETA ŁUCZYŃSKA *** \\ * Institute of Computer Science \\ Jagiellonian University, ul. Łojasiewicza 6, 30-438 Cracow, Poland \\ e-mail:jacek.smietanski@ii.uj.edu.pl \\ ** Department of Automatics \\ AGH University of Science and Technology, al. Mickiewicza 30, 30-059 Cracow, Poland \\ e-mail: rtadeagh.edu.pl \\ *** Department of Diagnostic Radiology \\ Centre of Oncology, ul. Garncarska 11, 31-115 Cracow, Poland \\ e-mail: dgn.ela@interia.pl
}

\begin{abstract}
The analysis of prostate images is one of the most complex tasks in medical images interpretation. It is sometimes very difficult to detect early prostate cancer using currently available diagnostic methods. But the examination based on perfusion computed tomography (p-CT) may avoid such problems even in particularly difficult cases. However, the lack of computational methods useful in the interpretation of perfusion prostate images makes it unreliable because the diagnosis depends mainly on the doctor's individual opinion and experience. In this paper some methods of automatic analysis of prostate perfusion tomographic images are presented and discussed. Some of the presented methods are adopted from papers of other researchers, and some are elaborated by the authors. This presentation of the method and algorithms is important, but it is not the master scope of the paper. The main purpose of this study is computational (deterministic and independent) verification of the usefulness of the $\mathrm{p}-\mathrm{CT}$ technique in a specific case. It shows that it is possible to find computationally attainable properties of p-CT images which allow pointing out the cancerous lesion and can be used in computer aided medical diagnosis.
\end{abstract}

Keywords: prostate cancer, perfusion computed tomography, medical image analysis, pattern recognition.

\section{Introduction}

Prostate cancer $(\mathrm{PCa})$ is one of the most common malignancies among men (ACS, 2009; NCR, 2009). In the last years there has still been observed a growth in the number of registered cases. And although it is partially connected with better and better diagnostic methods and increased knowledge among patients (resulting in better detectability of this type of cancer), there is no doubt that $\mathrm{PCa}$ is a serious medical and social problem.

Early detection of $\mathrm{PCa}$ is a key to survival. Unfortunately, routine medical tests like measuring blood concentration of prostate specific antigen (PSA), digital rectal examination (DRE), transrectal ultrasound (TRUS), and biopsy often fail (Hricak et al., 2007; Roscigno et al., 2004; Selley et al., 1997). For example, on TRUS, can- cer lesions can be hypoechoic, hyperechoic or even isoechoic (Daehnert et al., 1986; Norberg et al., 1997; SudołSzopińska and Szopiński, 2005). In view of this, there is an obvious need for other diagnostic methods which could manage this problem in some cases which are too difficult for standard (above mentioned) methods.

There are many studies of new techniques which could address this problem, including, for example, the EPCA test (Bradford et al., 2006; Leman et al., 2007). Perfusion computed tomography (p-CT) is also one of these methods (still under investigation). This method allows evaluating the parameters of perfusion such as blood flow (BF), blood volume (BV), mean transit time (MTT), permeability surface (PS) in specified areas of prostate (ROI-region of interest) (Cenic et al., 2000; Wintermark et al., 2001). 
Nowadays the p-CT examination is used mainly in the diagnosis of brain acute stroke (Miles and Griffiths, 2003; Hartel et al., 2006; Hoeffner et al., 2004; Rosenberg et al., 2004), but the usefulness of this method has also been tested on other organs (Miles and Griffiths, 2003; Blomley et al., 1993; Dugdale and Miles, 1999; Dziubińska et al., 2006; Fukuya et al., 1995; Groell et al., 2001; Sahani et al., 2005; Wolfkiel et al., 1987; Zhang and Kono, 1997), including prostate (Henderson et al., 2003; Ives et al., 2005; Łuczyńska et al., 2008; Prando and Wallace, 2000). Its application to detecting cancerous lesions is based on documented evidence of the creation of new blood vessels in tumor (angiogenesis) (Charlesworth and Harris, 2006; Miles, 1999; 2002). Although prostate is not highly vascularized, it is supposed that p-CT can indicate these suspicious areas also in this gland.

The purpose of this study is computational verification of usefulness of the p-CT technique in a specific case, described in the paper (Euczyńska et al., 2006). In that case, diagnostics correctly pointed cancerous lesions on the $\mathrm{p}$-CT image, while on TRUS there were no visible suspicious regions. However, that indication was founded only on visual assessment, so it can be considered undeterministic and unreliable.

\section{Images}

A 60-year-old patient was examined at the Oncology Center in Cracow because of an increasing PSA level. The pCT examination was performed with a 16-slice CT scanner (GE Ligh Speed). The perfusion level was measured during repeated scans of the minor pelvis at $120 \mathrm{kVp}$ and $200 \mathrm{mAs}$. The scans were started about $10 \mathrm{~s}$ after administering of $50 \mathrm{ml}$ of non-ionic contrast medium $(370 \mathrm{mgI} / \mathrm{ml})$ at the rate of $5 \mathrm{ml} / \mathrm{s}$ and lasted $50 \mathrm{~s}$. The total width of the diagnosed area was $20 \mathrm{~mm}$.

Parametric maps (BF, BV, MTT and PS) were drawn using the CT Perfusion 3 application on the Advantage Workstation at three levels (conventionally base, middle and apex) of the gland.

In order to perform computational analysis, only the area of prostate was selected from the acquired images. The images, originally coded with pseudocolor, where blue symbolizes the area with minimal and redwith maximal perfusion, were transformed into a 31-tone grayscale using the LUT table (Tadeusiewicz and Korohoda, 1997), where 0 means maximal visible perfusion (red area in pseudocolor) and 30-no perfusion. (Fig. 1)

Figure 2 presents parametric maps of the prostate (coded with pseudocolor) at the level at which pathological lesions were confirmed (Fig. 3). In this work only the image of Fig. 2(a), which represents the parameter BF, is selected for further analysis.

\section{Co-occurrence matrices}

For automatic description of the texture of particular regions on the analyzed p-CT image, the so-called cooccurrence matrices (Haralick et al., 1973) were selected. There are many other texture analysis methods, but these are most universal and their potential is greatest.

Let $I: \mathbb{Z}^{2} \supset D \rightarrow G=\left\{1, \ldots, N_{g}\right\}$ (where $\mathbb{Z}$ denotes set of integers) be a two-dimensional discrete image with $N_{g}$ gray levels. For the given image $I$, we define the co-occurrence matrix (GLCM):

$$
\begin{array}{r}
P_{0}(i, j \mid d, \theta)=\#\{k, l \in D: I(k)=i, I(l)=j, \\
\|k-l\|=d, \angle(k-l)=\theta\}
\end{array}
$$

or, in a normalized version,

$$
\begin{aligned}
& P(i, j \mid d, \theta) \\
& =\frac{\#\{k, l \in D: I(k)=i, I(l)=j,\|k-l\|=d, \angle(k-l)=\theta\}}{\#\{m, n \in D:\|m-n\|=d, \angle(m-n)=\theta\}},
\end{aligned}
$$

where $i, j \in G$ stand for gray levels of points $k$ and $l$, respectively, $\angle(k-l)$ is the angle between vector $\overrightarrow{k l}$ and axis $\overrightarrow{0 X}, d$ represents the distance between $k$ and $l, \theta$ is the direction of co-occurrence, $\# X$ represents the power (number of elements) of set $X$.

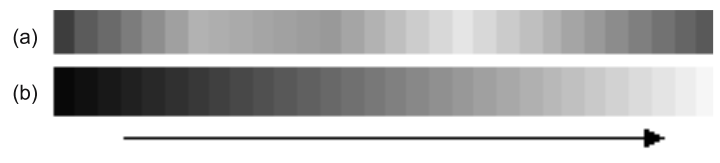

Fig. 1. Pseudocolor (a), grayscale after transformation (b). The arrow shows a rise in the perfusion values.

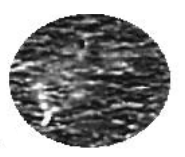

(a)

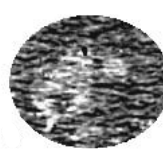

(b)

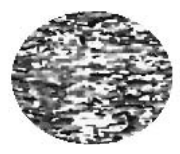

(c)

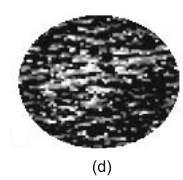

(d)
Fig. 2. p-CT images of the prostate: blood flow (BF) (a), blood volume (BV) (b), mean transit time (MTT) (c), permeability surface (PS) (d).

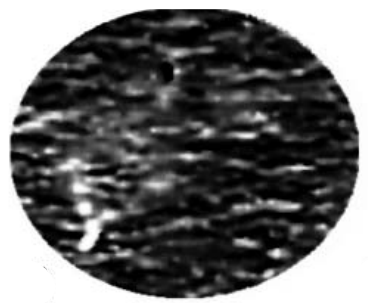

(a)

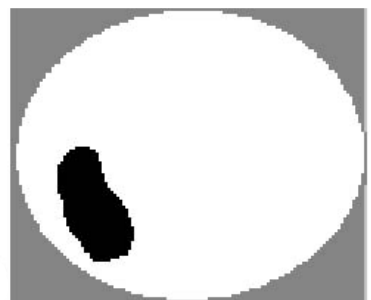

(b)
Fig. 3. Analyzed image (a) and cancerous area (b)—shown in black. 
Table 1. Coefficients of GLCM

\begin{tabular}{|c|c|c|c|}
\hline no. & name & abbr. & value \\
\hline$f_{1}$ & energy & ENE & $f_{1}=\sum_{i, j} P(i, j)^{2}$ \\
\hline$f_{2}$ & entropy & ENT & $f_{2}=-\sum_{i, j} P(i, j) \log P(i, j)$ \\
\hline$f_{3}$ & homogeneity & IDM & $f_{3}=\sum_{i, j} \frac{1}{1+(i-j)^{2}} P(i, j)$ \\
\hline$f_{4}$ & inertia & $\mathrm{CON}$ & $f_{4}=\sum_{i, j}(i-j)^{2} P(i, j)$ \\
\hline$f_{5}$ & correlation & COR & $f_{5}=-\sum_{i, j} \frac{\left(i-\mu_{x}\right)\left(j-\mu_{y}\right)}{\sigma_{x} \sigma_{y}} P(i, j)$ \\
\hline$f_{6}$ & variance & VAR & $f_{6}=\sum_{i, j}\left(i+j-\mu_{x}-\mu_{y}\right)^{2} P(i, j)$ \\
\hline$f_{7}$ & shade & SHA & $f_{7}=\sum_{i, j}\left(i+j-\mu_{x}-\mu_{y}\right)^{3} P(i, j)$ \\
\hline$f_{8}$ & prominence & PRO & $f_{8}=\sum_{i, j}\left(i+j-\mu_{x}-\mu_{y}\right)^{4} P(i, j)$ \\
\hline$f_{9}$ & sum average & SA & $f_{9}=\sum_{i=2}^{2 N_{g}} i P_{x+y}(i)$ \\
\hline$f_{10}$ & sum entropy & SE & $f_{10}=-\sum_{i=2}^{2 N_{g}} P_{x+y}(i) \log P_{x+y}(i)$ \\
\hline$f_{11}$ & sum variance & SV & $f_{11}=-\sum_{i=2}^{2 N_{g}}\left(i-f_{9}\right)^{2} P_{x+y}(i)$ \\
\hline$f_{12}$ & difference average & DA & $f_{12}=\sum_{i=0}^{N_{g}-1} i P_{x-y}(i)$ \\
\hline$f_{13}$ & difference entropy & $\mathrm{DE}$ & $f_{13}=-\sum_{i=0}^{N_{g}-1} P_{x-y}(i) \log P_{x-y}(i)$ \\
\hline$f_{14}$ & difference variance & DV & $f_{14}=-\sum_{i=0}^{N_{g}-1}\left(i-f_{12}\right)^{2} P_{x-y}(i)$ \\
\hline$f_{15}$ & information measure & IMC1 & $f_{15}=\frac{f_{2}-\mathrm{HXY}_{1}}{\max (\mathrm{HX}, \mathrm{HY})}$ \\
\hline$f_{16}$ & coefficient of variation & COV & $f_{16}=\frac{\sigma(P(i, j))}{\mu(P(i, j))}$ \\
\hline$f_{17}$ & peak transition probability & MAX & $f_{17}=\max (P(i, j))$ \\
\hline$f_{18}$ & diagonal variance & DIAV & $f_{18}=\sigma^{2}(P(i, j))$ \\
\hline$f_{19}$ & diagonal moment & DIAM & $f_{19}=\sum_{i, j}\left(\frac{1}{2}|i-j| P(i, j)\right)^{\frac{1}{2}}$ \\
\hline$f_{20}$ & second diagonal moment & DSM & $f_{20}=\sum_{i, j} \frac{1}{2}|i-j| P(i, j)$ \\
\hline$f_{21}$ & triangular symmetry & TRS & $f_{21}=|P(i, j)-P(j, i)|$ \\
\hline \multicolumn{4}{|c|}{$\begin{array}{l}\text { Notation } \\
\mu_{x}=\sum_{i} i \sum_{j} P(i, j), \mu_{y}=\sum_{j} j \sum_{i} P(i, j), \\
\sigma_{x}=\sum_{i}\left(i-\mu_{x}\right)^{2} \sum_{j} P(i, j), \sigma_{y}=\sum_{j}\left(j-\mu_{y}\right)^{2} \sum_{i} P(i, j), \\
P_{x}(i)=\sum_{j} P(i, j), P_{y}(j)=\sum_{i} P(i, j), \\
P_{x+y}(k)=\sum_{i, j: i+j=k} P(i, j), P_{x-y}(k)=\sum_{i, j:|i-j|=k} P(i, j), \\
\mathrm{HX} \text {-entropy } P_{x}(i), \mathrm{HY} \text {-entropy } P_{y}(j), H X Y_{1}=-\sum_{i, j} P(i, j) \log \left(P_{x}(i) P_{y}(j)\right)\end{array}$} \\
\hline
\end{tabular}


The GLCM allows us to evaluate a number of coefficients, which characterize the textures of the analyzed image. Table 1 shows the list of 21 coefficients used in our study.

\section{Results}

For the given image of Fig. 3 (a) transformed to grayscale we evaluated the first-order statistics calculated directly from the image histogram (Table 2). The mean in a healthy area is smaller than in a cancerous one but the variance is very high in both cases. Therefore, the analysis based only on the first-order statistics of the ROI considered (see below) may not be sufficient (Table 3, Fig. 6).

The ROIs covering the analyzed image were rectangular in shape, 10 pixels wide and 20 pixels high. Each consecutive ROI was selected 10 pixels apart the previous

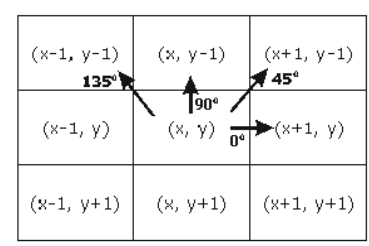

(a)

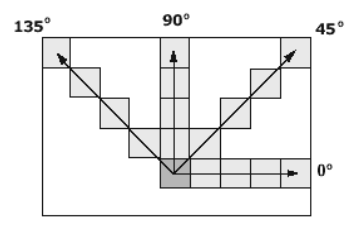

(b)
Fig. 4. Nearest neighborhood of the point (x,y) and directions of co-occurrence (a), co-occurrence can be considered also for a greater distance between pairs of points (b).

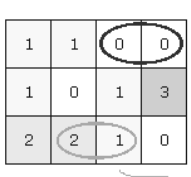

(a)

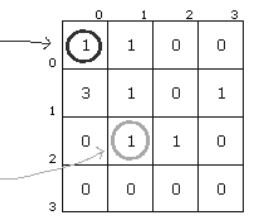

(b)

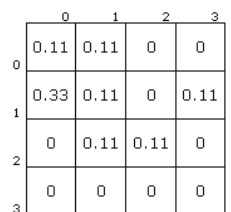

(c)
Fig. 5. Example of GLCM: source image with four gray levels (a), illustration of counting co-occurrences for $d=$ $1, \theta=0^{\circ}$ (b), GLCM, $d=1, \theta=0^{\circ}$ (counted cooccurrences are divided by the number of all pairs of points considered (here 9) (c). In this example the values were rounded to two decimal places.

Table 3. Statistics of the ROI. For each ROI considered, the mean and median were evaluated. In the table we show the minimum and the maximum of those valuesseparately for ROIs covering the healthy region and separetely for ROIs in the cancerous area.

\begin{tabular}{|l|c|c|c|c|}
\hline \multirow{2}{*}{ ROI } & \multicolumn{2}{|c|}{ mean } & \multicolumn{2}{c|}{ median } \\
\cline { 2 - 5 } & $\min$ & $\max$ & $\min$ & $\max$ \\
\hline \hline healthy region & 2.33 & 9.92 & 1 & 10 \\
\hline cancerous region & 8.17 & 16.42 & 4 & 16 \\
\hline
\end{tabular}

one. Those where less than half of the pixels covered the area of prostate were missed. Each ROI was classified according to the pattern shown in Fig. 3 (b). There were 88 ROIs at all: 82 healthy and six cancerous (Fig. 7). For each ROI, normalized GLCM matrices (see Eqn. (2)) and coefficients were evaluated.

There were calculated 21 coefficients (Table 1) for each GLCM characterized by distance $d$ in the range from 1 to 9 , and angle $\theta$ with values $0^{\circ}, 45^{\circ}, 90^{\circ}, 135^{\circ}$, and also $d$ in the range from 10 to 19 and $\theta=90^{\circ}$. So it was the 966-dimensional feature space. The resulting values for each feature were analyzed in order to eliminate outliers and normalized. The distribution of each feature was equalized using the ladder of powers method (Tukey, 1977; Velleman and Hoaglin, 1981) (see Eqn. 3) with $\gamma \in(0,2]$

$\operatorname{error}(\gamma)=\sum_{c=1,2}\left(\int_{x}\left[\operatorname{cdf}\left\{x_{c}^{\gamma}\right\}-\Phi\left\{\overline{x_{c}^{\gamma}}, \operatorname{var}\left(x_{c}^{\gamma}\right)\right\}\right]^{2}\right)$

where $c=\{1,2\}$ represents classification, $\operatorname{cdf}\left(x_{c}^{\gamma}\right), \overline{x_{c}^{\gamma}}$, $\operatorname{var}\left(x_{c}^{\gamma}\right)$ stand for the distribution function, mean and variance of empirical distribution for class $c$, respectively, $\Phi\left(\mu, \sigma^{2}\right)$ is a normal distribution function with mean $\mu$ and variance $\sigma^{2}$.

We were looking for $\gamma_{\mathrm{opt}}$ which minimizes the function $\operatorname{error}(\gamma)$ :

$$
\gamma_{\mathrm{opt}}=\min _{\gamma} \operatorname{error}(\gamma)
$$

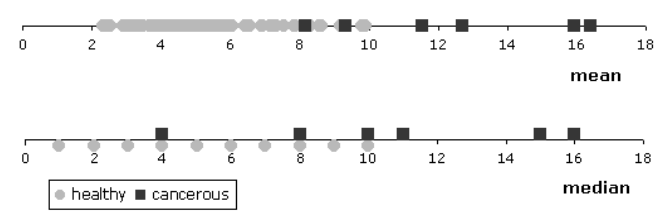

Fig. 6. Mean and median of the analyzed ROIs. Light circleshealthy regions, dark squares_cancerous regions.

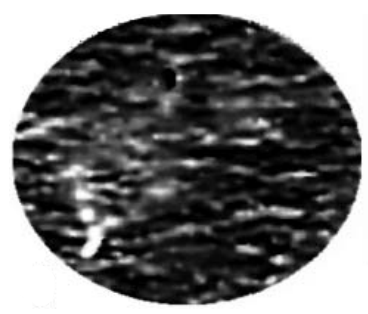

(a)

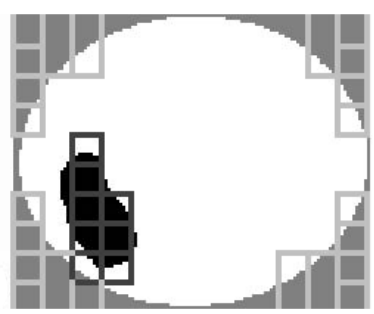

(b)
Fig. 7. Analyzed image (a) and pattern (b). The cancerous area is shown in black. Also ROIs classified as cancerous (dark gray) and not analyzed (light gray) are shown on the pattern. Other ROIs, which are not shown, were classified as a healthy area. 
Table 2. First-order statistics of the analysed image.

\begin{tabular}{|l|c|c|c|c|c|c|}
\hline parameter & mean & median & variance & std.dev. & skewness & curtosis \\
\hline \hline all image & 5.72 & 4 & 33.98 & 5.83 & 1.63 & 2.88 \\
\hline healthy area & 5.20 & 4 & 26.15 & 5.11 & 1.52 & 2.51 \\
\hline cancerous area & 12.47 & 11 & 85.21 & 9.23 & 0.47 & -0.92 \\
\hline
\end{tabular}

Features where $\operatorname{error}\left(\gamma_{\mathrm{opt}}\right) \geq 1$ were excluded from further analysis. For each of the remaining features, the Bhattacharyya measure (Bhattacharyya, 1943) was used for the normal distribution:

$$
J=\frac{1}{4} \frac{\left(\mu_{1}-\mu_{2}\right)^{2}}{\sigma_{1}^{2}+\sigma_{2}^{2}}+\frac{1}{2} \ln \left(\frac{\sigma_{1}^{2}+\sigma_{2}^{2}}{2 \sigma_{1} \sigma_{2}}\right),
$$

where $\mu_{1}, \mu_{2}$ are means, $\sigma_{1}, \sigma_{2}$ are standard deviations for Classes 1 and 2, respectively. Below, in Table 4 we present a list of the best discriminating properties. As is shown, the best results were produced for the diagonal moment $\left(f_{19}\right)$ and various $d$ and $\theta$. It should be noted that diagonal directions $\theta=45^{\circ}$ and $\theta=135^{\circ}$ did not occur in any of the best ten features.

As can be remarked, the above-mentioned consideration is limited to the indication of a single individually best discriminating feature (Fig. 8). It should be observed that, in spite of these limitations, it is possible to indicate such features which individually have the ability to distinguish a healthy and a cancerous area (Fig. 9). However, it is not a universal rule-even for features with a large distance between classes, sometimes these areas cannot be separated (Fig. 10). In such cases it can be helpful to increase the dimension of the feature space (Fig.11).

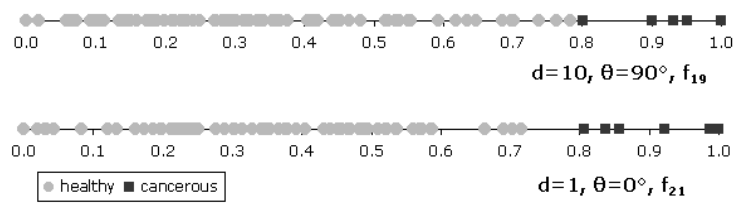

Fig. 9. Classification of two example parameters from Table 4. It is possible to point out the border value, where lower values suggest a healthy area and higher values mean susceptibility of a cancerous lesion.

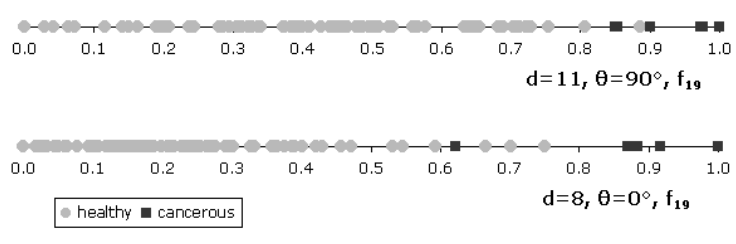

Fig. 10. In spite of high discriminant power in these examples we cannot point out the border value.

\section{Conclusion}

In this paper it was shown that it is possible to select such parameters of an image which are deterministic and independent of a personal assessment. Our results confirm the usefulness of the p-CT method applied to PCa diagnosis in the analyzed case. Of course, it is obvious that only one case cannot be generalized, but in this study the potential of this method can be seen.

At the Oncology Center in Cracow the p-CT method is used to examine other patients. Thanks to that it will be possible to verify the usefulness of the proposed algorithm. In further work the authors will also expand research to other perfusion parameters to determine the effectiveness of each one.

\section{References}

ACS (2009). Estimated new cancer cases and deaths by sex, US, http: / / www. cancer.org/downloads/stt/ cff2009_estcd_3.pdf.

Bhattacharyya, A. (1943). On a measure of divergence between two statistical populations defined by their probability distributions, Bulletin of the Calcutta Mathematical Society 35: 99-110.

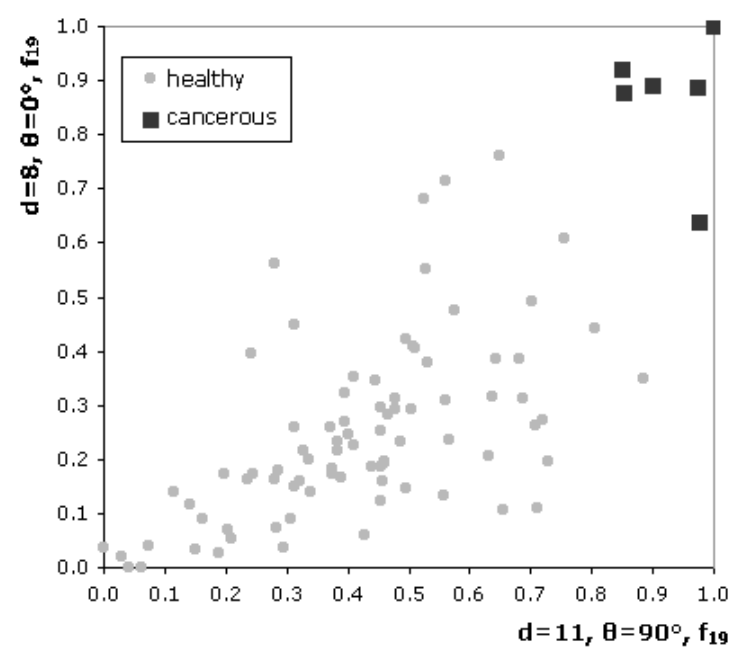

Fig. 11. Improvement of discriminant power after increasing the feature space dimension. Features shown in Fig. 10, which were useless individually, are very good to separate the classes in a two-dimensional space. 
Table 4. List of ten features with the best discriminant power.

\begin{tabular}{|c|c|c|c|}
\hline displacement $\mathrm{d}$ & angle $\theta$ & coefficient & Bhattacharyya measure \\
\hline \hline 8 & 0 & diagonal moment $\left(f_{19}\right)$ & 2.494145 \\
\hline 10 & 90 & diagonal moment $\left(f_{19}\right)$ & 2.441980 \\
\hline 1 & 0 & triangular symmetry $\left(f_{21}\right)$ & 2.181827 \\
\hline 3 & 90 & prominence $\left(f_{8}\right)$ & 2.074908 \\
\hline 3 & 0 & variance $\left(f_{6}\right)$ & 2.051128 \\
\hline 6 & 90 & sum entropy $\left(f_{10}\right)$ & 1.962097 \\
\hline 11 & 90 & diagonal moment $\left(f_{19}\right)$ & 1.860219 \\
\hline 4 & 90 & prominence $\left(f_{8}\right)$ & 1.843247 \\
\hline 11 & 90 & second diagonal moment $\left(f_{20}\right)$ & 1.839980 \\
\hline 4 & 0 & sum entropy $\left(f_{10}\right)$ & 1.836315 \\
\hline
\end{tabular}

Blomley, M., Coulden, R., Bufkin, C., Lipton, M. and Dawson, P. (1993). Contrast bolus dynamic computed tomography for the measurement of solid organ perfusion, Investigative Radiology 28(suppl 5): S72-S77.

Bradford, T., Tomlins, S., Wang, X. and Chinnaiyan, A. (2006). Molecular markers of prostate cancer, Urologic Oncology 24(6): 538-551.

Cenic, A., Nabavi, D., Craen, R., Gelb, A. and Lee, T.-Y. (2000). A CT method to measure hemodynamics in brain tumors: Validation and application of cerebral blood flow maps, American Journal of Neuroradiology 21(3): 462-470.

Charlesworth, P. and Harris, A. (2006). Mechanisms of disease: Angiogenesis in urologic malignancies, nature clinical practice, Urology 3(3): 157-169.

Daehnert, W., Hamper, U., Eggleston, J., Walsh, P. and Sanders, R. (1986). Prostatic evaluation by transrectal sonography with histopathologic correlation: The echopenic appearance of early carcinoma, Radiology 158(1): 97-102.

Dugdale, P. and Miles, K. (1999). Hepatic metastases: The value of quantitative assessment of contrast enhancement on computed tomography, European Journal of Radiology 30(3): 206-213.

Dziubińska, M., Basiak, M., Konopka, M., Hartel, M., PilchKowalczyk, J. and Sassiadek, M. (2006). Role of perfusion imaging in computed tomography in diagnostics of cerebral gliomas, Neurologia i Neurochirurgia Polska 40(1): 51-56, (in Polish).

Fukuya, T., Honda, H., Hayahi, T., Kaneko, K., Tateshi, Y., Ro, T., Maehara, Y., Tanaka, M., Tsuneyoshi, M. and Masuda, K. (1995). Lymph-node metastases: Efficacy of detection with helical CT in patients with gastric cancer, Radiology 197(3): 705-711.

Groell, R., Doerfler, O., Schaffler, G. and Habermann, W. (2001). Contrast-enhanced helical CT of the head and neck. Improved conspicuity of squamous cell carcinoma on delayed scans, American Journal of Roentgelogy 176(6): 1571-1575.
Haralick, R., Shanmugam, K. and Dinstein, I. (1973). Textural features for image classification, IEEE Transactions on Systems, Man and Cybernetics 3(6): 610-621.

Hartel, M., Dziubińska-Basiak, M., Konopka, M., Basiak, M., Salam, B. and M., S. (2006). Complex diagnostic imaging of acute ischemic stroke-Case study, Udar Mózgu 8(2): 81-86, (in Polish).

Henderson, E., Milosevic, M., Haider, M. and Yeung, I. (2003). Functional CT imaging of prostate cancer, Physics in Medicine and Biology 38(18): 3085-3100.

Hoeffner, E., Case, I., Jain, R., Gujar, S., Shah, G., Deveikis, J., Carlos, R., Thompson, B., Harrigan, M. and Mukherji, S. (2004). Cerebral perfusion CT: Technique and clinical applications, Radiology 231(3): 632-644.

Hricak, H., Choyke, P., Eberhardt, S., Leibel, S. and Scardino, P. (2007). Imaging prostate cancer. A multidisciplinary perspective, Radiology 243(1): 28-53.

Ives, E., Burke, M., Edmonds, P., Gomella, L. and Halpern, E. (2005). Quantitative computed tomography perfusion of prostate cancer: Correlation with whole-mount pathology, Clinical Prostate Cancer 4(2): 109-112.

Leman, E., Cannon, G., Trock, B., Sokoll, L., Chan, D., Mangold, L., Partin, A. and Getzenberg, R. (2007). Epca-2: A highly specific serum marker for prostate cancer, Urology 69(4): 714-720.

Łuczyńska, E., A. J. S. J., Stelmach, A., Jaszczyński, J., Hartel, M. and Konopka, M. (2006). Perfusion of prostate cancer: Correlation between p-CT and whole-mount pathologyCase report, Polish Journal of Radiology 71(4): 70-73.

Łuczyńska, E., Anioł, J., Stelmach, A. and Jaszczyński, J. (2008). The value of perfusion CT in evaluating locoregional staging in post-radical prostatectomy patients with elevated serum PSA level, Polish Journal of Radiology 73(2): 13-17.

Miles, K. (1999). Tumour angiogenesis and its relation to contrast enhancement on computed tomography: A review, European Journal of Radiology 30(3): 198-205. 


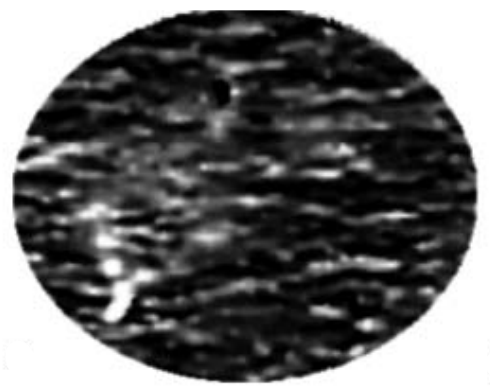

(a)

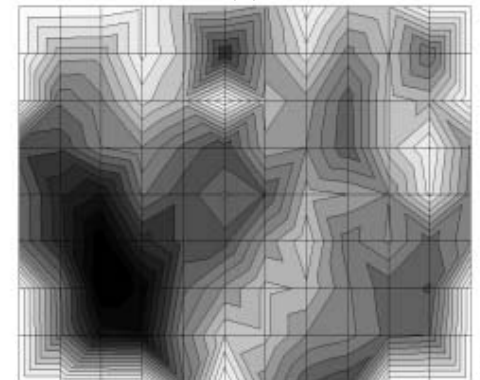

(d)

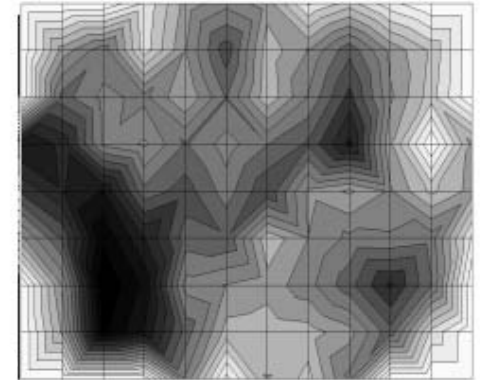

(g)

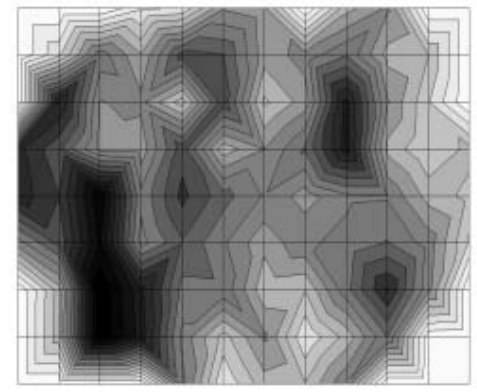

(j)

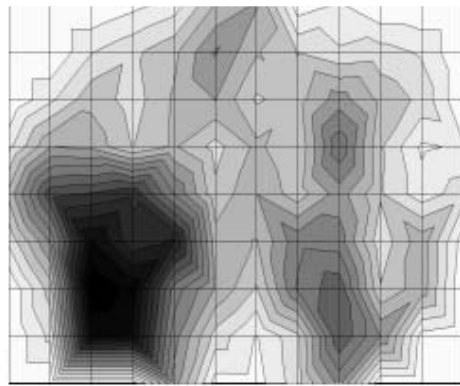

(b)

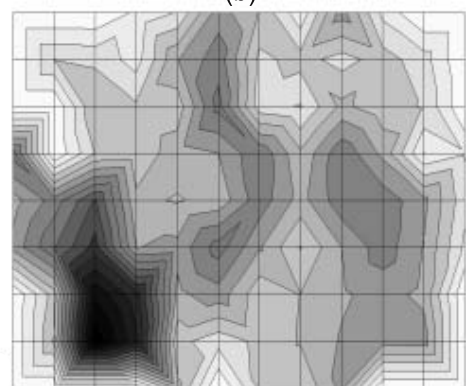

(e)

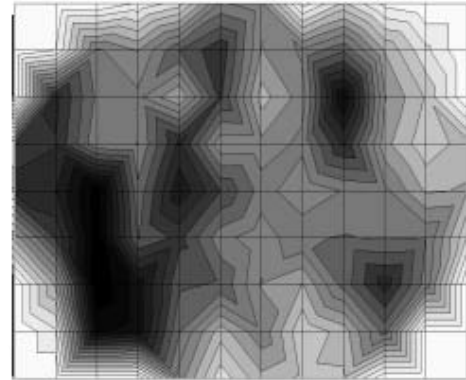

(h)

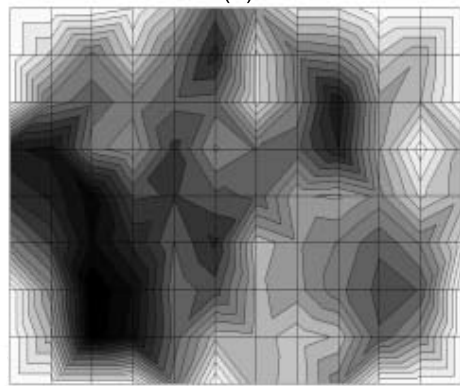

(k)

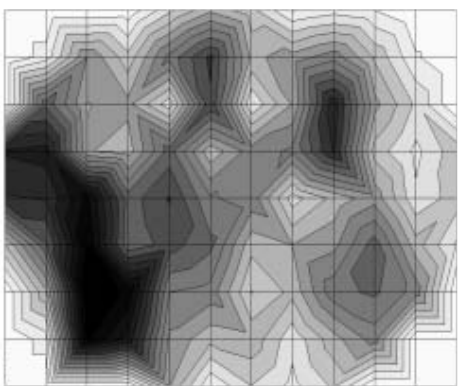

(c)

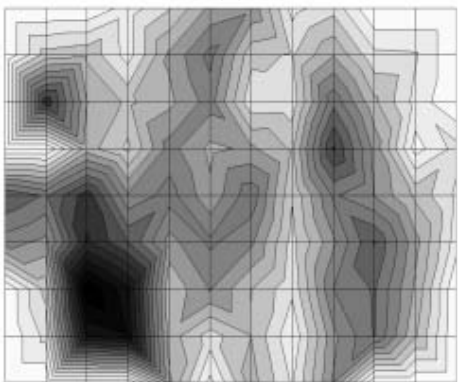

(f)

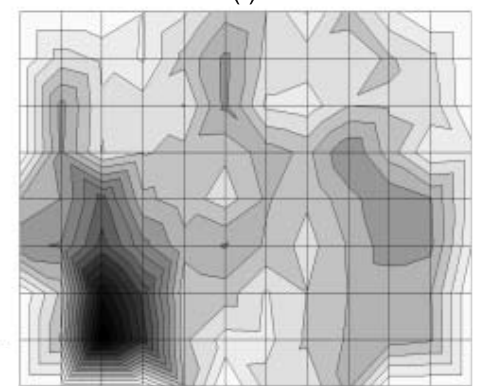

(I)

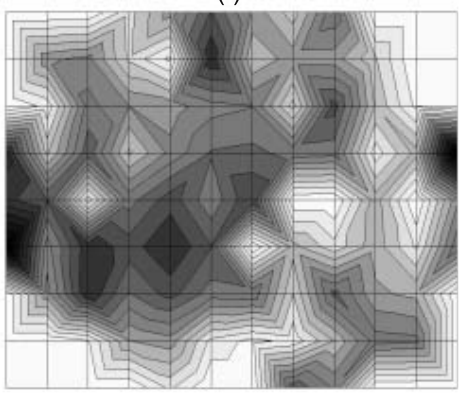

(I)

Fig. 8. Analyzed image (a), illustration of the best discriminating features (b-k), and of the feature with no discriminant power (l). $d=8, \theta=0^{\circ}, f_{19}(\mathrm{~b}), d=10, \theta=90^{\circ}, f_{19}(\mathrm{c}), d=1, \theta=0^{\circ}, f_{21}(\mathrm{~d}), d=3, \theta=90^{\circ}, f_{8}$ (e), $d=3, \theta=0^{\circ}, f_{6}$ (f), $d=6, \theta=90^{\circ}, f_{10}(\mathrm{~g}), d=11, \theta=90^{\circ}, f_{19}(\mathrm{~h}), d=4, \theta=90^{\circ}, f_{8}(\mathrm{i}), d=11, \theta=90^{\circ}, f_{20}(\mathrm{j}), d=4, \theta=0^{\circ}, f_{10}(\mathrm{k})$, $d=8, \theta=45^{\circ}, f_{15}(1)$.

Miles, K. (2002). Functional computed tomography in oncology, European Journal of Cancer 38(16): 2079-2084.

Miles, K. and Griffiths, M. (2003). Perfusion CT: A worthwhile enhancement?, British Journal of Radiology 76(904): 220 231.

NCR (2009). Number of new cancer cases by site and age groups, http://85.128.14.124/krn/english/ liczba_zach_rozp/default.asp.
Norberg, M., Egevad, L., Holmberg, P., Sparen, P., Norlén, J. and Busch, C. (1997). The sextant protocol for ultrasoundguided core biopsies of the prostate underestimates the presence of cancer, Urology 50(4): 562-566.

Prando, A. and Wallace, S. (2000). Helical CT of prostate cancer: Early clinical experience, American Journal of Roentgenology 175(2): 343-346.

Roscigno, M., Scattoni, V., Bertini, R., Pasta, A., Montorsi, 
F. and Rigatti, P. (2004). Diagnosis of prostate cancer. State of the art, Italian Journal of Urology and Nephrology 56(2): 123-145.

Rosenberg, R., Wojtek, P., Konopka, M., Pieniążek, P., Bogusz, I. and M., S. (2004). Clinical applications of perfusion computed tomography, diffusion-weighted magnetic resonance and perfusion-weighted magnetic resonance imaging in detection of early lesions in ischemic stroke, Udar мózgu 6(2): 71-78, (in Polish).

Sahani, D., Kalva, S., Hamberg, L., Hahn, P., Willett, C., Saini, S., Mueller, P. and Ty, L. (2005). Assessing tumor perfusion and treatment response in rectal cancer with multisection CT: Initial observations, Radiology 234(3): 785-792.

Selley, S., Donovan, J., Faulkner, A., Coast, J. and D., G. (1997). Diagnosis, management and screening of early localized prostate cancer, Health Technology Assessment 1(2): 1-96.

Sudoł-Szopińska, I. and Szopiński, T. (2005). The USG Diagnostics of Prostate Gland, Praktyczna Ultrasonografia, Warszawa/Zamość, (in Polish).

Tadeusiewicz, R. and Korohoda, P. (1997). Computer Analysis and Image Processing, Wydawnictwo Fundacji Postępu Telekomunikacji, Cracow, (in Polish).

Tukey, J. (1977). Exploratory Data Analysis, Addison-Wesley, Reading, MA.

Velleman, P. and Hoaglin, D. (1981). Applications, Basics, and Computing of Exploratory Data Analysis, Duxbury Press, Boston, MA.

Wintermark, M., Maeder, P., Thiran, J.-P., Schnyder, P. and Meuli, R. (2001). Quantitative assessment of regional cerebral blood flows by perfusion CT studies at low injection rates: A critical review of the underlying theoretical models, European Radiology 11(7): 1220-1230.

Wolfkiel, C., Ferguson, J., Chomka, E., Law, W., Labin, I., Tenzer, M., Booker, M. and Brundage, B. (1987). Measurement of myocardial blood flow by ultrafast computed tomography, Circulation 76(6): 1262-1273.

Zhang, M. and Kono, M. (1997). Solitary pulmonary nodules: Evaluation of blood flow patterns with dynamic CT, Radiology 205(2): 471-478.

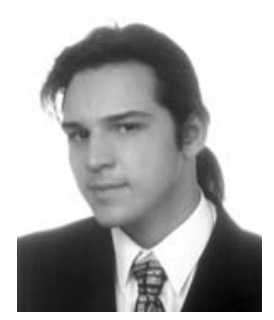

Jacek Śmietański received the M.Sc. degree in computer science from Jagiellonian University, and the Ph.D. degree in biomedical engineering from the AGH University of Science and Technology in 2003 and 2009, respectively. He has also studied biotechnology at Jagiellonian University. From 2003 to 2009, he held the position of an assistant lecturer at the Faculty of Pharmacy, Jagiellonian University Medical College. Currently he is an employee of the Jagiellonian University Institute of Computer Science. His research interests cover the area of image processing and artificial intelligence. Particularly, he tries to solve important medical and biological problems using advanced computational techniques. He is an author of some publications, including articles in international journals, conference proceedings, as well as academic textbooks. He has also prepared an e-learning web-page for pharmacy students.

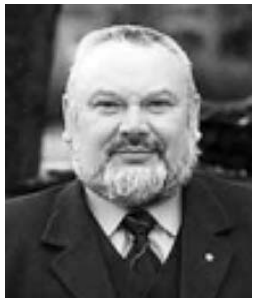

Ryszard Tadeusiewicz studied at the Electrical Engineering Department of the University of Mining and Metallurgy in Cracow (Poland), from which he graduated (with honors) in 1971 Additionally, after receiving his degree in automatic control engineering, he studied at the Faculty of Medicine at the Medical Academy in Cracow and undertook studies in the field of mathematical and computer methods in economics. He has written over 600 scientific papers, published in prestigious Polish and foreign scientific journals as well as numerous conference presentations, both national and international. Professor Tadeusiewicz has also authored over 70 scientific monographs and books, including highly popular textbooks (which have had many editions). He has been the supervisor of 56 doctoral theses and a reviewer of more than 200 doctoral theses. In 2007, Polish scientists elected him a chapter chair of the Poland Section of the IEEE Computational Intelligence Society

Elżbieta Luczyńska received a Ph.D. in radiology and imaging diag nostic in 2003 and a diploma of medical sciences in 1992 from Collegium Medicum, Jagiellonian University in Cracow, Poland. Since 1995, she has been with the Skłodowska-Curie Oncology Centre, Cracow Department, and since 2005, she has been the head of the Radiology and Image Diagnostics Department, Oncology Centre in Cracow. Her main interests include the evaluation of diagnostic modalities in cancer detection. She is an author and co-author of 29 publications, a co-author of two books, an author of six reports presented during international conferences and 60 reports presented during national conferences. Since 2007 she has given lectures in English on oncologic radiology for Norwegian and American students in the framework of a school for foreigners of Collegium Medicum at Jagiellonian University. She is a member of the Polish Medical Radiology Society and the European Radiology Society, the Polish Oncology Society and the Surgical Oncology Society.

Received: 24 March 2009

Revised: 18 August 2009 